\title{
Evaluation of a novel real-time fluorescent polymerase chain reaction assay for high-risk human papilloma virus DNA genotypes in cytological cervical screening
}

\author{
JIAOYING CHENG, MEILU BIAN, XIAO CONG, AIPING SUN, MIN LI, LI MA, YING CHEN and JUN LIU
}

Department of Gynecology and Obstetrics, China-Japan Friendship Hospital, Beijing 100029, P.R. China

Received September 17, 2012; Accepted October 18, 2012

DOI: $10.3892 / \mathrm{br} .2012 .49$

\begin{abstract}
It has been confirmed that detection of high-risk human papillomavirus (HR HPV) DNA is useful in cervical cancer (CC) screening. Recently, a new real-time fluorescent polymerase chain reaction (PCR) assay was developed to detect HR HPV. This assay can synchronize nucleic acid amplification and testing using specific primers for 13 types of HR HPV genomes, combined with specific TaqMan fluorescent marker probe techniques through the fluorescence automatic PCR instrument. Furthermore, it uses TaqGold ${ }^{\mathrm{TM}}$ DNA polymerase, which minimizes the amount of non-specific amplification and increases the sensitivity of the assay. The aim of this study was to evaluate the analytical and clinical performance of the real-time fluorescent PCR assay in CC screening, compared to the Qiagen Hybrid Capture ${ }^{\circledR}$ II High-Risk HPV DNA test ${ }^{\circledR}$ (HC II). In total, 1,252 cervical specimens were collected from women between 19 and 71 years of age. The specimens were examined with three different assays, real-time fluorescent PCR assay and HC II for HR HPV detection combined with liquid-based cytology. Women with cytological abnormalities or HR HPV-positive results underwent colposcopy and cervical biopsy. This study demonstrated good overall agreement between HC II and real-time fluorescent PCR assay (overall agreement, 92.25\%; Cohen's $\kappa=0.814$ ). For the detection of high-grade cervical intraepithelial neoplasias (CIN) and CC, the sensitivity of HC II and real-time fluorescent PCR was 94.48 and $92.82 \%$, respectively, and the negative predictive value was 98.85 and $98.54 \%$, respectively. High HR HPV infection rate of the high-grade CIN and $\mathrm{CC}$ group was detected $(\mathrm{P}<0.05)$. In conclusion, real-time fluorescent PCR assay provides similar results compared to the HC II test for HR HPV detection and could be used in CC screening in clinic.
\end{abstract}

Correspondence to: Dr Meilu Bian, Department of Gynecology and Obstetrics, China-Japan Friendship Hospital, 2 Yinghua Dongjie, Hepingli, Beijing 100029, P.R. China

E-mail: bianmeilu@hotmail.com

Key words: human papillomavirus, real-time fluorescent polymerase chain reaction, hybrid capture II, cervical cancer

\section{Introduction}

Cervical cancer (CC) is the second most frequent malignant tumor disease among the various types of female cancer and threatens female life expectancy and health. There have been 495,000 new cases recently worldwide, with the age bracket of patients shifting into younger age groups. Additionally, patients (15-35\%) with high-grade cervical intraepithelial neoplasia (CIN) or CC are often not detected at first screening.

A causal link between human papillomavirus (HPV) infection and $\mathrm{CC}$ has been established. A large number of HPV genotypes has been identified (1), and the HPV strains are divided into high- (HR) and low-risk (LR) categories on the basis of their association with cervical lesions. HR HPV, especially 13 HR HPV genomes (i.e., genotypes 16, 18, 31, 33, $35,39,45,51,52,56,58,59$ and 68$)$, are more frequently found in pre-malignant or malignant tumors. Infection by HR HPV has been demonstrated in $\sim 100 \%$ of CC. Clear scientific evidence indicates that screening based on validated tests for the DNA of oncogenic HPV as primary examination as well as the application of an appropriate protocol is more effective compared to screening based on cytology in preventing invasive types of cancer of the uterine cervix (2). Cytological analysis combined with HR HPV examination raises the detection rate of $\mathrm{CC}$, and is able to reduce $\geq$ grade 3 cervical intraepithelial neoplasia at 5 years (3). Furthermore, HPV testing is an important ancillary diagnostic tool for distinguishing which women are at risk to progress to the squamous intraepithelial lesion (SIL) stage, decreasing the number of colposcopy referrals and follow-up tests (4). As a result, the detection of HR HPV in cervical samples constitutes one of the most important steps in improving the efficacy of cervical carcinoma screening programs and in triaging patients with ambiguous or borderline cervical smears. An increasing number of studies has confirmed the effectiveness of HR HPV analysis in CC screening programs (5-8). The Hybrid Capture $^{\circledR}$ II High-Risk HPV DNA test ${ }^{\circledR}$ (HC II) was approved by the U.S. Food and Drug Administration in CC screening in women aged $\geq 30$ years (9), and has been widely used worldwide. Recently, a standardized PCR-based technique, the HPV real-time fluorescent PCR assay (Kaipu Biochemistry Co., Ltd., Wenzhou, China), was commercialized for the detection of the 13 HR HPV genotypes. This novel assay uses 
amplification of target DNA (L1 gene) by PCR and nucleic acid hybridization for the detection of HR HPV genotypes in cervical cells collected into a transport medium. This assay could be used in clinical diagnostic laboratories, however, limited data are currently available on its performance and reliability. Specifically, no data exist on its comparison with the HC II test.

The aim of this study was to examine the performance of two commercially available tests, HC II test and real-time fluorescent PCR assay, and to detect the presence of HR HPV in cervical samples of women who attended the Department of Gynecology and Obstetrics of China-Japan Friendship Hospital (Beijing, China) for CC screening. This study provides clinicians with an additional, lower-cost choice in $\mathrm{CC}$ screening compared to the HC II test.

\section{Materials and methods}

Study population. From July 15th, 2009 to January 10th, 2011, 1,252 women underwent $\mathrm{CC}$ screening at the Department of Gynecology and Obstetrics of China-Japan Friendship Hospital (Beijing, China). Written informed consent was obtained from all the patients. The study was approved by the ethics committee of China-Japan Friendship Hospital. The patient mean age was $40.8 \pm 9.5$ years (range, 19-71); $90 \%$ of patients were aged $\geq 30$ years and $86 \%$ of them were 30-65 years. Specimens for the liquid-based cytology test (LCT), HC II test and real-time fluorescent PCR assay were collected from the women during their visit. Colposcopy was performed when $\geq 1$ of the results of these three tests was abnormal, and a histological sample was taken when a lesion was identified. Women exhibiting acute genital inflammation were excluded from this study.

Sample collection. The women were asked to avoid vaginal douching, vaginal drug administration 3 days prior to their visit to the hospital as well as sexual activity $24 \mathrm{~h}$ prior to their visit. During the collection of cervical cells, we firstly cleaned and polished the cervix with cotton pledgets. Secondly, cervical samples were obtained with cervix brushes rotated for three times on any days of the menstrual cycle, except those during menstrual bleeding. Thirdly, we removed samples from the brushes and placed them into sample tubes containing preserving fluid and marked the name of the patient as well as the date. Specimens were preserved for $<24 \mathrm{~h}$ at $2-8^{\circ} \mathrm{C}$ or for 30 days at $-20^{\circ} \mathrm{C}$. Repeated specimen thawing was avoided.

$L C T$. Smears were stained using the AutoCyte Prep staining machine and observed by professional gynecology-pathology doctors with cytological diagnosis aptitude. Cytologic diagnosis was performed according to the Cervical Cytology Criteria of the 2001 Bethesda System of the International Cancer Society. According to this system, abnormalities of cervical squamous epithelium include atypical squamous cells (ASC), low- (LSIL) and high-grade squamous intraepithelial lesions (HSIL), as well as squamous cell carcinomas (SCC).

HPV DNA testing using the HC II test. HR HPV DNA testing was performed using the automated HC II test (Qiagen, Gaithersburg, MD, USA). HC II test is a sandwich capture molecular hybridization assay that utilizes chemiluminescent detection to provide a semi-quantitative result. The test was calibrated to detect $\sim 5,000$ genome $/ \mathrm{ml}$ equivalents of target $\mathrm{HPV}$, represented by a Relative Light Unit (RLU) measurement greater than or equal to the cut-off value calculated in each run by a series of standards. A measurement less than the cut-off value was scored as negative. The samples were analyzed for the presence of HR HPV types 16, 18, 31, 33, 35, 39, 45, 51, 52, 56, 58, 59 and 68. Three positive and 3 negative controls (provided by the manufacturer) were included in each run.

HPV DNA testing using real-time fluorescent PCR assay. This assay included: i) DNA extraction, involving the release of HPV and cellular DNA by lysing cervical specimens under denaturing conditions at elevated temperatures in the presence of protease. The assay did not require DNA purification. ii) PCR amplification and hybridization reaction, involving the use of biotinylated primers in order to define a sequence of $\sim 150$ bp in length within the polymorphic L1 region of the HPV genome. The primers, pooled in the same PCR Master mix, were designed to amplify viral DNA from the same 13 HR HPV types included in the HC II test. Capture probes representing regions internal to the amplified sequences were used to identify HPV of human DNA. PCR amplification and hybridization reaction were performed using the Roche LightCycler ${ }^{\circledR}$. Thirty cytological samples were detected simultaneously, and each time negative and positive controls were established. Following the reaction, Roche LightCycler ${ }^{\circledR}$ computer was used to calculate the cycle threshold (CT) of each sample. CT was the cycle number of each reaction flourescence signal that reached the setting threshold. iii) Result interpretation involved the baseline and threshold exceeding culmination of the amplication curve of the negative control, while the $\mathrm{CT}$ value did not indicate detection (blank). Evaluation of response effectiveness depended on the blank negative control and a CT of $\leq 36$ in the positive control. When the CT value of the samples was $\leq 40$, samples were evaluated as positive, while samples were considered negative with a $\mathrm{CT}>40$. According to the manufacturer's instructions, this test was able to detect the genotypes of the 13 HR HPV types at 5,000 copies/ml.

Colposcopy and biopsy. Colposcopic examination of the cervix was performed in women with either HR HPV-positive results or cytological abnormalities, or both. Negative cytological results and the two HR HPV DNA tests used predicted a low risk of cervical neoplasia. A histological sample was obtained when a lesion was identified, and 4 histological samples were obtained at $3^{\circ}, 6^{\circ}, 9^{\circ}$ and $12^{\circ}$ of the normal transformation zone. Endocervical canal curettage was performed when colposcopic examination was unsatisfactory. The patients with high-grade lesions underwent loop electrosurgical excision procedure (LEEP), cold knife conization (CKC) or hysterectomy for further diagnosis and treatment. The most severe diagnosis was determined as the final histopathological diagnosis. The diagnostic criteria of high-grade lesions were defined as equal to or more severe than CINII (CINII+).

Statistical analysis. Sensitivity, specificity and accuracy of the real-time fluorescent PCR assay, the HC II test and the LCT 
Table I. Comparison between the outcomes of 1,252 samples examined using HC II test and real-time fluorescent PCR assay.

Real-time fluorescent PCR assay outcome, $n$

HC II test outcome

HPV-positive

HPV-negative

Total, $\mathrm{n}$

\begin{tabular}{lrrr}
\hline HPV-positive & 321 & 59 & 380 \\
HPV-negative & 38 & 834 & 872 \\
Total & 359 & 893 & 1,252 \\
\hline
\end{tabular}

HC II, Hybrid Capture ${ }^{\circledR}$ II High-Risk HPV DNA test ${ }^{\circledR}$; HPV, human papillomavirus; PCR, polymerase chain reaction.

were determined against the presumed HPV status based on the combination of the two HPV tests used and histological pathological results. The $95 \%$ confidence intervals (CI) were calculated using Fisher's exact test. Agreement between the two HPV tests used was assessed by Cohen's $\kappa$, with values of 0.00-0.40 indicating poor, 0.40-0.75 fair and 0.75-1.00 excellent agreement. The $\chi^{2}$ test was performed for group comparisons. All the tests were two-sided, and $\mathrm{P}<0.05$ was considered to indicate a statistically significant difference.

\section{Results}

Outcomes of 1,252 specimens examined using HC II test and real-time fluorescent PCR assay. The comparison between HC II test and real-time fluorescent PCR assay of 1,252 specimens is shown in Table I. Among the 1,252 women studied, the two tests gave similar results for 1,155 samples, with an overall level of agreement of $92.25 \%$ (Cohen's $\kappa=0.814$ ) indicating excellent agreement. However, the examination of 97 samples using the two tests gave discordant results. HPV genotyping of these samples are to be performed in future studies. Assuming that samples with positive results examined using the two tests were infected by HR HPV, and that samples with negative results were HR HPV-negative or infected by LR HPV, 'conditional' sensitivity and specificity can be evaluated for the two tests used.

Correlation between histopathological outcomes and HPV test. There were 477 patients who underwent colposcopic examination and biopsy. Histopathological results were considered to be the 'gold standard'. Sherman et al (10) found that the cumulative incidence rate was only $0.79 \%$, when CIN or CC and negative HPV cases were not detected using cervical cytological examination, while CIN and cancer cases were not identified in these histopathology samples. On these grounds, women with normal LCT results and negative results from the two HR HPV DNA tests used were classified into the "negative' group (women without CIN or CC). As shown in Fig. 1, 1,015 specimens were histopathologically negative (normal or inflammation). Among these samples, 182 (17.93\%) were positive using the HC II test, while 162 (15.96\%) were positive using the real-time fluorescent PCR assay. Additionally, 56 samples were diagnosed as CINI using histopathological examination of which 27 (48.21\%) were positive using the HC II test and $29(51.79 \%)$ were positive using the real-time fluorescent PCR assay. Fifty-five samples were diagnosed as CINII using histo-

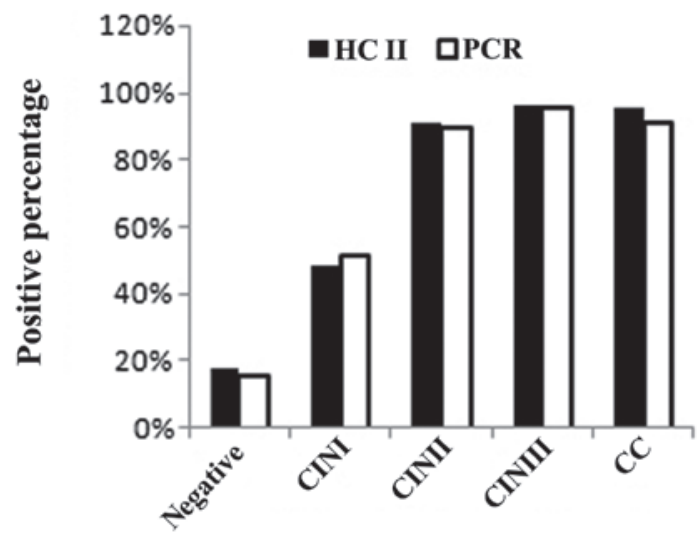

Histopathological diagnosis

Figure 1. HR HPV infection in various cervical specimens. HC II, Hybrid Capture $^{\circledR}$ II High-Risk HPV DNA test ${ }^{\circledR}$; PCR, polymerase chain reaction; CINI-III, cervical intraepithelial neoplasia I-III; CC, cervical cancer.

pathological examination of which $50(90.90 \%)$ were positive using the HC II test, while $49(89.09 \%)$ were positive using the real-time fluorescent PCR assay. A further 104 samples were diagnosed as CINIII using histopathological examination of which $100(96.15 \%)$ were positive using the HC II test, while 99 (95.19\%) were positive using the real-time fluorescent PCR assay. Furthermore, 22 samples were diagnosed as CC using histopathological examination of which 21 (95.45\%) were positive using the HC II test, while 20 (90.90\%) were positive using the real-time fluorescent PCR assay. We compared the negative and positive histopathological groups, where the HR HPV infection rate was 19.51 and $94.48 \%$, respectively, when samples were examined using the HC II test. A statistically significant difference between two groups was observed $\left(\chi^{2}=411.57 ; \mathrm{P}<0.05\right)$.

Due to the fact that CINII and CINIII patients did not undergo the CINI morphology stage, the difficulty to distinguish HPV infection from the changes of CINI at cytopathological appearance under a colposcope, and the significant proportion of samples that would undergo spontaneous regression, we classified histopathological results of CINII, CINIII and CC into the positive group in this study, and normal or inflammation and CINI samples into the negative group. Sensitivity and specificity were also calculated at this point. The correlation between the two HPV tests used and histopathological outcomes is shown in Table II. 
Table II. Correlation between HPV tests and histopathological outcomes.

\begin{tabular}{lccccrrr}
\hline & \multicolumn{2}{c}{ HC II test outcome, $\mathrm{n}$} & & \multicolumn{2}{c}{ Real-time fluorescent PCR assay outcome, $\mathrm{n}$} & \\
\cline { 2 - 3 } Histopathological outcome & HPV-positive & HPV-negative & & HPV-positive & HPV-negative & Total, $\mathrm{n}$ \\
\hline HPV-positive & 171 & 10 & & 168 & 13 & 181 \\
HPV-negative & 209 & 862 & & 191 & 880 & 1,071 \\
Total & 380 & 872 & & 359 & 893 & 1,252 \\
\hline
\end{tabular}

HPV, human papillomavirus; HC II, Hybrid Capture ${ }^{\circledR}$ II High-Risk HPV DNA test ${ }^{\circledR}$; PCR, polymerase chain reaction.

In this study, there were 181 patients with CINII+, $94.48 \%$ of whom were positive using the HC II test and $92.82 \%$ of whom were positive using the real-time fluorescent PCR assay. Out of the 1,071 samples of the negative histopathology group, there were $862(80.49 \%)$ negative samples using the HC II test and $880(82.17 \%)$ negative samples using the real-time fluorescent PCR assay. Out of the 872 negative samples using the $\mathrm{HC}$ II test, there were 862 patients without CINII+, indicating a negative predictive value of $98.85 \%$. Out of the 893 negative samples using the real-time fluorescent PCR assay, there were 880 patients without CINII+, indicating a negative predictive value of $98.54 \%$.

\section{Discussion}

A causal link between HR HPV infection and CC has been established. According to Sharma et al (11), conducting a population-based HPV survey targeting women $>35$ years of age would be sufficient to estimate the $\mathrm{CC}$ risk in a certain country. This study has shown the difference of the HR HPV infection rate between the normal or inflammation group and the CINII+ group $\left(\chi^{2}=411.57 ; \mathrm{P}<0.05\right)$.

Numerous methods can be utilized to detect HR HPV infection. The HC II test has been considered the best method to screen for CC. Real-time fluorescent PCR is a novel assay developed to use TaqGold ${ }^{\mathrm{TM}}$ DNA polymerase, which minimizes the amount of non-specific amplification and increases the sensitivity of the assay. Furthermore, this PCR protocol is based on a 5'-exonuclease assay and real-time detection of fluorescence accumulation. Fluorescence release during each amplification cycle is directly proportional to the amount of amplicon generated. Therefore, it is considered to be an accurate method for estimating viral load (?).

The aim of this study was to compare: i) the performance of two commercially available assays (HC II test and real-time fluorescent PCR assay) which cover the same HR HPV genotypes for the detection of the $13 \mathrm{HR}$ HPV types as well as ii) the sensitivity, specificity and accuracy of the two tests for CINII+ screening in a group of 1,252 women. The overall correlation of the two tests was $92.25 \%$, with a Cohen's $\kappa$ value of 0.814 , indicating good agreement. However, 97 samples yielded different results ( $7.75 \%$ of all samples): 59 were positive using the HC II test and negative using the real-time fluorescent PCR assay. Of these 59 women, 10 were diagnosed as CINII+, while 38 were positive using the real-time fluorescent PCR assay and negative using the HC II test. Of these 38 women,
8 patients were diagnosed as CINII+. Although neither of the two tests was able to detect all of the CINII+ cases during CC screening, LCT and HR HPV tests may be combined in order to increase accuracy.

Previous studies $(12,13)$ have demonstrated that the Digene HC II assay may have cross-reactivity with LR HPV types, known to cause cytological abnormalities that do not progress to cancer. Subsequent studies are to investigate whether the real-time PCR HPV test is likely to recognize the LR HPV type as HR HPV. Regardless of the discordant samples, the two HPV tests used showed a good correlation and yielded comparable results. In clinical practice, when choosing the most appropriate HPV test, gynecologists should consider whether the test has good negative predictive value and a satisfactory sensitivity of detecting clinical lesions, and whether or not the test is reproducible and has unitive standard.

This study suggests that the real-time fluorescent PCR assay possesses the above characteristics. The sensitivity of this assay detecting CINII+ is $~ 92.82 \%$, and the negative predictive value is $\sim 98.54 \%$. An additional characteristic of the real-time fluorescent PCR assay constitutes its excellent quality combined with its reasonable price, expending only a half of the HC II test. As a result, it could reduce the expense of $\mathrm{CC}$ screening in developing countries. However, due to the fact that it has the ability to amplify shorter fragments, it is considered to have a higher analytical sensitivity and a lower clinical specificity, as well as to be adaptable for less well-preserved specimens.

In conclusion, the present study suggests that real-time fluorescent PCR assay and HC II test are easily implemented in a clinical laboratory and that they provide comparable results. Although this study found a number of modest differences between the two tests, a fact which will be further studied in future studies, it provides gynecologists with an additional, low-cost choice in CC screening.

\section{References}

1. Munoz N, Bosch FX, de Sanjose S, Herrero R, Castellsaque X, Shah KV, Snijders PJ and Meijer CJ; International Agency for Research on Cancer Multicenter Cervical Cancer Study Group: Epidemiologic classification of human papilomavirus types associated with cervical cancer. N Engl J Med 348: 518-527, 2003.

2. Ronco G, Biggeri A, Confortini M, et al: Health technology assessment report: HPV DNA based primary screening for cervical cancer precursors. Epidemiol Prev 36: e1-e72, 2012 (In Italian). 
3. Budenholzer B: ACP Journal Club. Adding HPV testing to cytology screening reduced $\geq$ grade 3 cervical intraepithelial neoplasia at 5 years. Ann Intern Med 157: JC2-JC7, 2012.

4. Leinonen M, Nieminen P, Kotaniemi-Talonen L, Malila N, Tarkkanen J, Laurila P and Anttila A: Age-specific evaluation of primary human papillomavirus screening vs. conventional cytology in a randomized setting. J Natl Cancer Inst 101: $1612-1623,2009$.

5. Davey DD and Zarbo RJ: Human papillomarivus testing - are you ready for a new era in cervical cancer screening? Arch Pathol Lab Med 127: 927-929, 2003.

6. Damasus-Awatai G and Freeman-Wang T: Human papillomavirus and cervical screening. Curr Opin Obstet Gynecol 15 : 473-477, 2003.

7. Petry KU, Menton S and Menton M, et al: Inclusion of HPV testing in routine cervical cancer screening for woman above 29 years in Germany: results for 8,466 patients. Br J Cancer 88: $1570-1577,2003$

8. Wright TC Jr, Schiffman M, Solomon D, et al: Interim guidance for the use of human papillomavirus DNA testing as an adjunct to cervical cytology for screening. Obstet Gynecol 103: 304-309, 2004
9. Saslow D, Runowicz CD, Solomon D, et al: American Cancer Society guideline for the early detection of cervical neoplasia and cancer. CA Cancer J Clin 52: 342-362, 2002.

10. Sherman ME, Lorincz AT, Scott DR, et al: Baseline cytology, human papillomavirus testing, and risk for cervical neoplasia: a 10-year cohort analysis. J Natl Cancer Inst 95: 46-52, 2003.

11. Sharma M, Bruni L, Diaz M, Castellsaqué X, de Sanjosé SD, Bosch FX and Kim JJ: Using HPV prevalence to predict cervical cancer incidence. Int J Cancer: Sep 11, 2012 (Epub ahead of print). doi: 10.1002/ijc.27835.

12. Castle PE, Schiffman M, Burk RD, et al: Restricted crossreactivity of hybrid capture 2 with nononcogenic human papillomavirus types. Cancer Epidemiol Biomarkers Prev 11: 1394-1399, 2002.

13. Poljak M, Marin IJ, Seme K and Vince A: Hybrid Capture II HPV test detects at least 15 human papillomavirus genotypes not included in its current high-risk probe cocktail. J Clin Virol 25: S89-S97, 2002. 